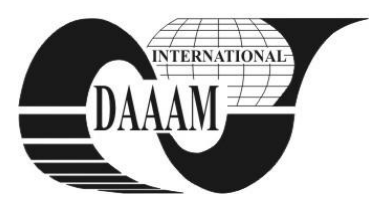

Annals of DAAAM for 2012 \& Proceedings of the 23rd International DAAAM Symposium, Volume 23, No.1, ISSN 2304-1382 ISBN 978-3-901509-91-9, CDROM version, Ed. B. Katalinic, Published by DAAAM International, Vienna, Austria, EU, 2012 Make Harmony between Technology and Nature, and Your Mind will Fly Free as a Bird Annals \& Proceedings of DAAAM International 2012

\title{
EXAMINATION OF THE QUALITY OF BARCODE REPRODUCTION
}

\author{
MARKOTIC, D[anijela]; PUCEKOVIC, N[ikolina] \& BATES, I[rena]
}

\begin{abstract}
Barcode is a kind of code in which the words, sentences or their parts, such as letters and numbers of plain text, are replaced by groups of letters or numbers depending on code replacements. Codes are used for securing confidential content (military, diplomatic, etc.). The basic theme of this paper is the functionality of barcodes after their printing on labels. For that reason, quality tests are performed with lines printed by means of two types of digital printing technologies on three different printing substrates. Contrast, blurriness and raggedness of lines were tested and compared on prints to see which digital technique and what kind of paper substrate were the most suitable for printing barcodes on labels. These three properties, among many others, define readability of bar codes. Today, digital printing is a very popular printing technique. Ink-jet and laser printing technique, also known as electrophotography, is used for printing simulated EAN bar codes.
\end{abstract}

Keywords: barcode, blurriness, contrast, raggedness

\section{INTRODUCTION}

In last few decades barcode technology has been successfully used for identification of products with minimal human intervention. It is used for gathering information about products as quickly as possible. A barcode symbol contains key information about the product [1]. To read information, codes have to be scanned. This is where most errors occur, and printing of barcodes is also often a source of error. If the barcode quality is higher, error probability is smaller and the time of scanning and reading code is faster [1].According to Mrotzek, the accuracy of the barcode symbols is influenced by printing, type of scanner and type of symbols [2].Barcodesconsistof a series ofthickand thinverticaldark andlightercolumns that areprinted onlabelsfor retail, business and commercialapplications, providing afast, accurate and effectivemethodof collecting andrecordingdata [3].

The measured line edge raggedness values provided by the recent research showed marked differences among the six different media types which were printed by inkjet technology. The plain paper produced an average edge raggedness of $20 \mu \mathrm{m}$, whereas glossy film produced raggedness of only about $8 \mu \mathrm{m}$. It was also found that the raggedness of the lines was highly correlated with the line orientation, horizontal vs. vertical [4].Other research clearly showed the difference between the images from different printers, but paper was ignored as an influential parameter, because the same paper was used for all the prints [5].

ISO 13660 standard is the first international standard whose task is to incorporate a wide range of properties that characterize the quality of printing. According to the standard, the properties that define quality of barcode, among other things, are reproduction of line edge blurriness, line edge raggedness and line contrast [4].

This paper presents analysis and comparison of two different line widths $(0.1 \mathrm{~mm}$ and $4 \mathrm{~mm})$ and their print reproduction measured in relation to the above mentioned three characteristics. The lines were printed on three different paper substrates with different degrees of smoothness, on white and yellow background. The prints were made by ink-jet and electrophotography.

The aim of this research was to determine which digital technology is the most suitable for printing barcodes on labels (simulated with different paper substrates and background colors).

\section{THEORETICAL PART}

Barcode columns must have sharp edges and high contrast so that scanner could read them. The highest readability of barcodes can be achieved with an opaque white background, which provides the highest reflections in free spaces between columns. If a transparent or colored paper substrate is used, background color should be in solid tone and in light color tone with maximum opacity in the areas that are used as background color for barcodes [6]. The best barcode reproduction is gained on smooth surfaces with a high range of accepting dyes. Rougher, textured and porous surfaces have a greater ability to print barcodes with voids and accepting dyes in free spaces between columns resulting in a reduced level of barcode readability [6]. Depending on the type of printing technique and used printer, there may be variations in printing reproduction of barcodes. Therefore, digital technologies used for this research are briefly described in the following...

Digital printing, unlike conventional, does not include any interphase, as the printing form and the information are printed directly on the paper. There are numerous advantages, including a very fast printing speed and printing of small runs at an affordable price offering personalized printing, since each printed sample is specifically prepared in the prepress and can contain specific data (e.g. user's name and address or barcode). There are two main techniques for digital printing, laser printing (electrophotography) and ink-jet. Besides these two techniques there are also magnetography and iconography [7].The main difference between electrophotography and ink-jet is that ink-jet does not use latent image carrier, ink is directly transferred to the printing surface, and only liquid toner is used [8]. 
ISO 13660:2001 is proposed by ISO/IEC JTC1/SC28 and is known as a method for measurement of image quality attributes. There are 14 different printed image's attributes that help analyse the printing defects, and they are categorized in two groups classified by their domain of appearance:

- Area attributes:

Darkness, background haze, graininess, mottle, extraneous marks and background voids.

- Character and line attributes:

Blurriness, raggedness, line width, darkness, character contrast, fill, extraneous marks, character field and background haze [5].

Many printing standards such as ISO 12647 are focusing on tone, color, resolution and contrast assessment in graphic arts images, while ISO 13660 provides quantitative assessment of fundamental printed attributes, such as area and line quality. This is very important because line and dot elements in any reproduction are the fundamental segments of the reproduction [5].

The blurriness is contrary characteristic of line edge sharpness. The measure of blurriness is the average distance between the inner and outer boundary edges. Raggedness is the appearance of geometric distortion of an edge from its ideal position. A ragged edge appears rough or wavy rather than smooth or straight. It is calculated with the standard deviation of the residuals from a line fitted to the edge threshold of the line. A good quality line may be described as the one having the least raggedness and sharp edges [5]. Contrast is the relationship between the darkness of a line segment, character image or other glyph image and its field. It is calculated using the standard formula:

$$
\text { Contrast }=\left(R_{\text {field }}-R_{\text {image }}\right) / R_{\text {field }}
$$

where $R_{\text {field }}$ is the mean reflectance factor of the surrounding character field and $R_{\text {image }}$ is the mean reflectance factor within the inner boundary edge of the image segment [9].

\section{EXPERIMENTAL PART}

Lines of different widths (from $0.1 \mathrm{~mm}$ to $4 \mathrm{~mm}$ ), simulating barcode columns, printed on the three different printing substrates were used for the measurements (see Fig. 1 and 2). The difference between these two samples, Fig. 1 and Fig. 2, is in the background color. The best readability is achieved with a combination of opaque black columns printed on opaque white background [6]. Therefore, Fig. 1 was printed in that combination. Fig. 2 consists of yellow background color, which is the most contrasting color that can be used as background color for barcodes [6]. It is also possible to use an orange, pink, peach and red background. Besides black color for barcode columns, it is possible to use dark blue and dark green but only on a white background [6].

The samples were printed on two digital printers using digital technique. HP Color LaserJet 2600n was used for electrophotography, and HP DeskJet F2480for ink-jet technique. Samples were measured with a digital microscope PIAS-II.

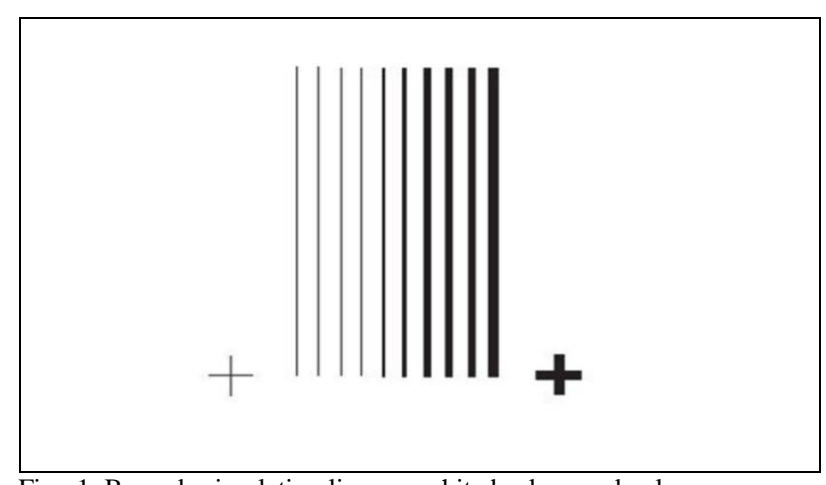

Fig. 1. Barcode simulation lines on white background color

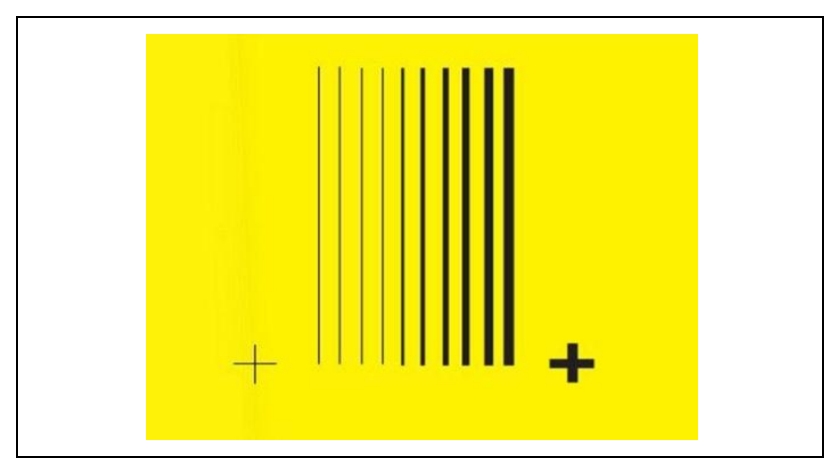

Fig. 2. Barcode simulation lines on yellow background color

HP Color LaserJet 2600n is famous for high acceptable output quality of text, images and graphics. The resolution that can be achieved is $600 \times 600 \mathrm{dpi}$, and the maximum print speed in color is 8 pages per minute.

HP DeskJet $F 2480$ inkjet multifunction printer is characterized by print resolution up to $4800 \times 1200 \mathrm{dpi}$ and the ability to print 16 colorpages per minute.

PIAS-II (Personal Image Analysis System) is a digital microscope that follows the ISO 13660 guidelines. It consists of two main parts, a measuring head which includes a digital camera, and two variable optical modules, of low and high resolution. High-resolution optical module is used for viewing small details like screening dots and variations in reproduction of lines, such as raggedness and blurriness. In the measurements required for this research, high-resolution optical module was used. Low-resolution module is useful for measuring printing malformations, such as uniformity and color banding [9].

The paper substrates with their grammage $\left(\mathrm{g} / \mathrm{m}^{2}\right)$ and smoothness values according to the Bekk method (sec) used in the measurements are listed in Table 1.

\begin{tabular}{|c|c|c|}
\hline $\begin{array}{l}\text { Paper } \\
\text { substrate }\end{array}$ & Grammage[g/m $\left.\mathbf{m}^{\mathbf{2}}\right]$ & $\begin{array}{c}\text { Smoothness } \\
\text { [sec; Bekk } \\
\text { method] }\end{array}$ \\
\hline PS 1 & 100 & 41.34 \\
\hline PS 2 & 100 & 271.88 \\
\hline PS 3 & 120 & 3.28 \\
\hline
\end{tabular}

The paper substrates were different with respect to smoothness range. Smoothness is a very important characteristic, because it impacts readability of barcodes. 
It is defined by the Bekk method, and the value of smoothness is given in seconds. As the value of seconds is higher, the paper surface is smoother.

After printing the samples on these substrates, the values of contrast, blurriness and raggedness of edge line were measured with PIAS-II digital microscope.

\section{RESULTS AND ANALYSIS}

The following Fig. $s$ show the difference in blurriness, raggedness and contrast between the widest (4 $\mathrm{mm})$ and narrowest line $(0.1 \mathrm{~mm})$.

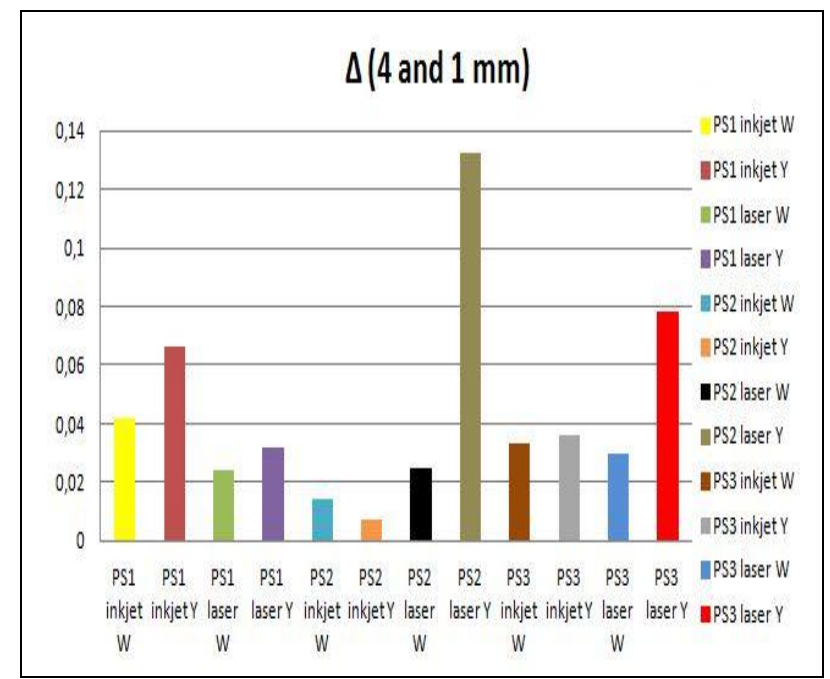

Fig. 3. Difference between 4 and $0.1 \mathrm{~mm}$ in blurriness

The analysis shown in Fig. 3 indicates that the difference between the widest and narrowest line was most pronounced for paper substrate 2 with yellow background color (paper with grammage of $100 \mathrm{~g} / \mathrm{m}^{2}$ and smoothness of $271.88 \mathrm{sec}$ ), where the sample was printed with laser printing. In this case the difference was 0.13 $\mathrm{mm}$. The minimal difference was obtained on same substrate with ink-jet printing on yellow background, where it amounted to only $0.007 \mathrm{~mm}$. Regardingto blurriness of line edge, the difference was the largest on yellow background, especially with laser printing.

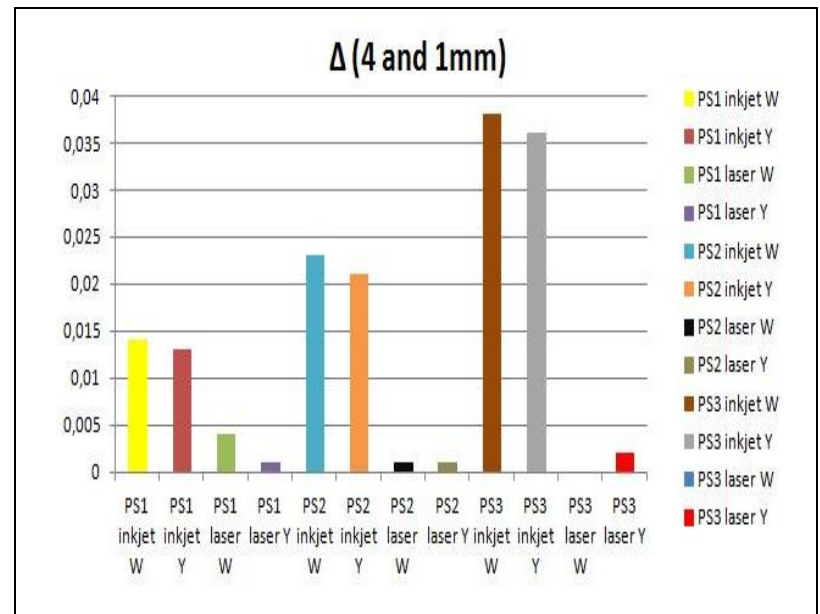

Fig. 4. Difference between 4 and $0.1 \mathrm{~mm}$ in raggedness

The analysis in Fig. 4 shows the maximum difference for paper substrate 3 with ink-jet printing technique. The maximum difference between $0.1 \mathrm{~mm}$ and $4 \mathrm{~mm}$ was found on paper substrate 1. Furthermore, there was no difference between the widest and narrowest line on paper substrate 3 with white background color (delta $=0$ ). Raggedness was bigger higher on white background whenever samples were printed with ink-jet technique.

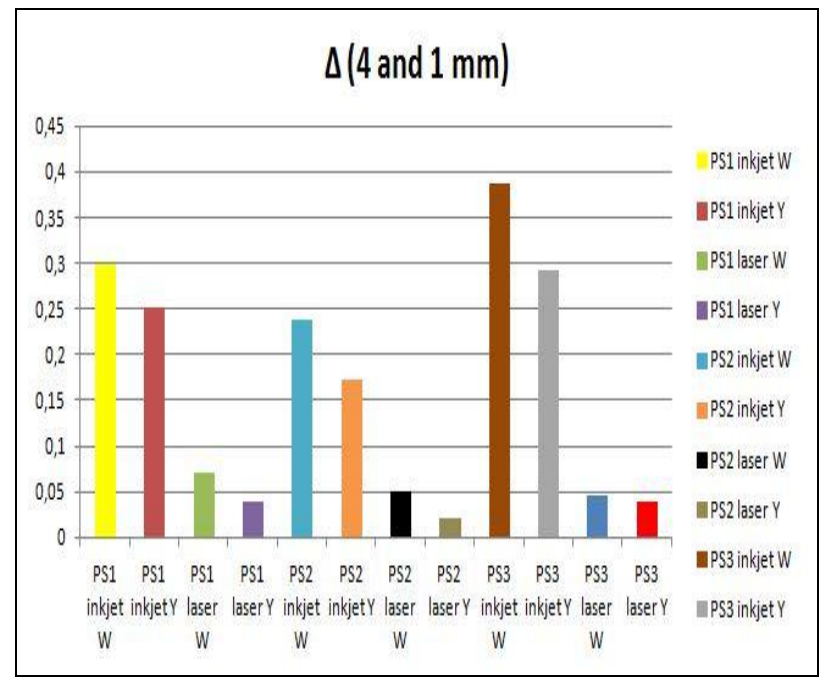

Fig. 5. Difference between 4 and $0.1 \mathrm{~mm}$ in contrast

There was a large difference in the contrast between the widest and narrowest line when the samples are printed with ink-jet printing technique (Fig. 5). The large differences are noticeable between ink-jet printed lines on white background and lines on yellow background. This difference ranges from 0.05 to $0.07 \mathrm{~mm}$. The sample printed with inkjet technique on paper substrate 3 with white background color showed the maximum difference. The minimum difference was established on paper substrate 2 with yellow background color, when laser printing was used.

\section{CONCLUSION}

Barcode is only effective if it can be read and/or scanned every time from the first attempt. Poor print quality of barcodes results in low range readability, imprecise form of symbols and customer's dissatisfaction. Acceptable barcode prints result in accuracy and consistency, high print quality and high contrast range.

Reproductions of the line width of $1 \mathrm{~mm}$ and $4 \mathrm{~mm}$ are presented in Fig. 6 and 7.

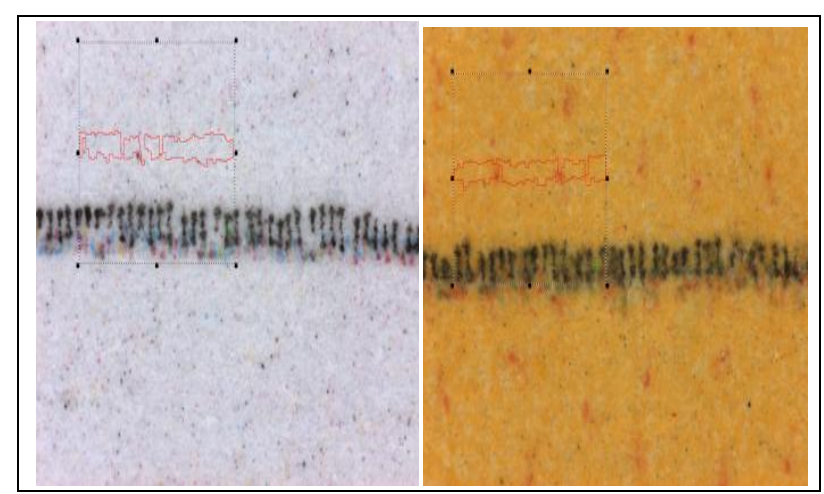

Fig. 6. Reproduction of a $0.1 \mathrm{~mm}$ wide line on paper substrate 1 on white (left) and yellow (right) background 


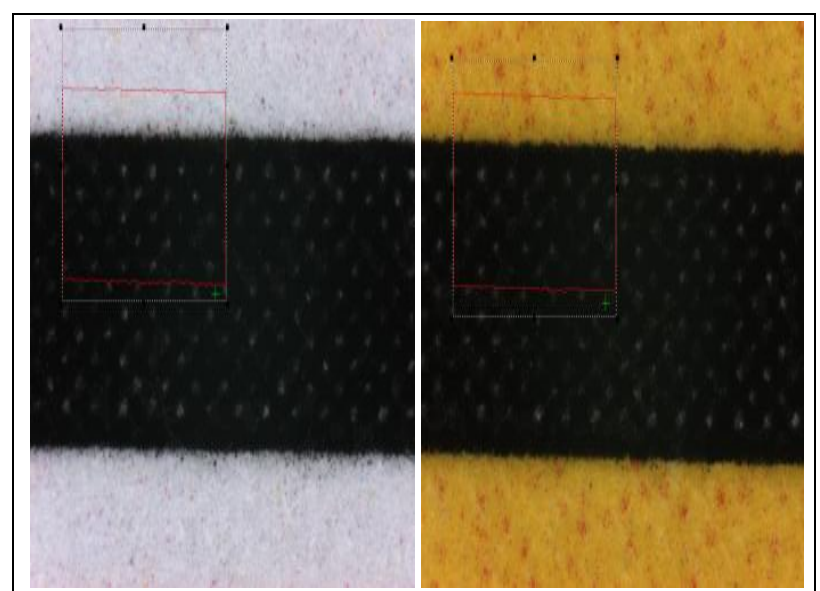

Fig. 7. Reproduction of a $4 \mathrm{~mm}$ wide line on paper substrate 1 in laser printing on white (left) and yellow (right) background

Regarding contrast and raggedness of line edge, the best paper substrate for printing barcodes was paper substrate 2, which was the smoothest.

Smoothness of the paper substrate impacted on two (contrast and raggedness) of the three examined line characteristics. Paper substrates 1 and 2 had the same grammage but different values for smoothness according to the Bekk method.

From this it can be concluded that smoothness impacts and determines raggedness of edge line. The higher the value of smoothness, the lower the raggedness of line edge is. In this research, the quality of barcode reproduction was measured through the difference between the widest and the narrowest simulated barcode line. The smaller this difference is, the higher the barcode quality on labels will be, and therefore easier and faster to read.

Besides printing substrates, printing technique is also very important for barcode quality. Laser printing is a better method for printing barcodes regarding contrast and raggedness of line edge because of smaller deformations in reproduction. The differences of examined line characteristics between $0.1 \mathrm{~mm}$ and $4 \mathrm{~mm}$ with laser printing are smaller than those with ink-jet printing. Printing with laser on white background regarding raggedness there was no differences between $0.1 \mathrm{~mm}$ and $4 \mathrm{~mm}$ line.

However, this conclusion is not applicable for blurriness of line edge. In that case, ink-jet printing is a better solution. Actually, the largest difference in blurriness between the widest and narrowest line occurs with laser printing.

Regarding background color, for twoexaminedline characteristics (contrast and raggedness), yellow background was a better solution than anunprinted paper. Concerning toblurriness of line edge, unprinted paper (white background) appeared to be a better solution.

It can be seen from the obtained results that it is essential to choose the proper printing substrate and printing technique when printing barcodes which are readable by the reading system.

Further measurements in connection with this research should include read rate of simulated barcode lines. Read rate is the ratio of the number of successful reads to the total number of read attempts, it is a measure of reliability of a barcode reading system [1]. A read rate of $100 \%$ is obviously a perfect, ideal number, number that we want to approach.

\section{REFERENCES}

[1] Billo R. E., Porter J.D., Mazumdar M., Brown S. J. (2003), Impact of Bar Code Print Quality on the Performance of HighSpeed Sortation Systems, Journal of Manufacturing Systems, Vol. 22/No. 4, (2003), page numbers (317-326)

[2] Mrotzek, G. (1998), Reliability of bar codes in a manufacturing environment."SCAN." The Data Capture Report (March 1998)

[3] Michael Fairley (2004.), Illustrated Encyclopedia of Labels and Label Technology, A labels \&Labeling Publication, Tarsus Publishing Ltd, ISBN 0-9547518-0-9, London

[4] Briggs J. C., Klein A. H., Ming-Kai Tse (1999) Applications of ISO-13660, A New International Standard for Objective Print Quality Evaluation, Paper presented at Japan Hardcopy '99 Imaging Society of Japan, July 21-23, 1999, Tokyo, Japan

[5] Dhopade A. (2009), Image quality assessment according to ISO 13660 and ISO 19751, Available from: http://cias.rit.edu/ gravure/tt/pdf/pc/TT9_image_quality_assessm ent.pdf, Accessed: 10th February 2011

[6] Flexographic Technical Association, Inc. (2003),Flexographic Image Reproduction Specifications \& Tolerances, Third Edition, Flexographic Technical Association, Inc. and the Foundation of Flexographic Technical Association, Inc., ISBN:0-9720474-4-1, Ronkonkoma.

[7] D. Bann (2007.) The all new print production Handbook, New York

[8] Kipphan H (2001.), Handbook of Print Media, Technologies and Production Methods, first ed., Springer, Heidelberg, Germany

[9] ISO/IEC 13660 Information Technology - Office Equipment Measurement of image quality attributes - Binary monochrome text and graphic images (2001). First edition, Reference number ISO/IEC 13660:2001(E). Geneva, Switzerland: International Organization for Standardization and International Electrotechnical Commission 